\title{
A Novel Hybrid Method for Classification of Tumor in Gene Expression Based Central Nervous System Microarray Data
}

\author{
W. Jai Singh ${ }^{1 *}$ and R. K. Kavitha ${ }^{2}$ \\ ${ }_{182}^{12}$ Assistant Professor (SRG), Department of Computer Applications \\ Kumaraguru College of Technology, Coimbatore, India
}

\section{ABSTRACT}

Nowadays, DNA microarray is widely used by researchers to predict cancer disease. In a microarray data, the presence of large number of features and instances makes it difficult to analyse and diagnose cancer. Hence, the selection of features is considered to be a vital task in classifying data. The phenotypical and genotypical behaviour of tumors in the human central nervous system poses a challenge in diagnosing and treating the disease. A clear description of the tumor is necessary to diagnose and treat the disease. Many feature selection methods can be used to identify the genes which are expressed differently in a microarray data. This research proposes a novel method of categorizing tumors present in Central Nervous System (CNS) with the help of DNA microarray gene representation present in the samples collected from patients. This research work aims to blend techniques like Support Vector Machine (SVM), Information Gain (IG) and Principal Component Analysis (PCA). Initially, Information Gain technique was used to select features followed by feature reduction with the help of principal component analysis. Finally, support vector machine was employed to classify the disease as cancer or not. This study was carried out with the popularly used CNS microarray dataset. It can be observed from the study results that the proposed approach proves to be effective in yielding a superior accuracy in classifying the disease with minimal number of genes.

\section{KEY WORDS: CLASSIFICATION, DIMENSIONALITY REDUCTION, FEATURE SELECTION, MICROARRAY, SUPPORT VECTOR} MACHINE.

\section{INTRODUCTION}

The tumor found in the human central nervous system can be an unusual cell growth in brain or the spinal cord tissues. CNS tumor which is a universal term comprises of one hundred and twenty individual types of tumors. It has been discussed in few studies that the identification and forecast of these tumors post a great challenge due its

\section{ARTICLE INFORMATION}

*Corresponding Author: jaisingh.w.mca@kct.ac.in

Received 15th Oct 2020 Accepted after revision 12th Dec 2020

Print ISSN: 0974-6455 Online ISSN: 2321-4007 CODEN: BBRCBA

Thomson Reuters ISI Web of Science Clarivate Analytics USA and Crossref Indexed Journal

\section{Clarivate
Analytics}

NAAS Journal Score 2020 (4.31) SJIF: 2020 (7.728)

A Society of Science and Nature Publication,

Bhopal India 2020. All rights reserved.

Online Contents Available at: http//www.bbrc.in/

Doi: http://dx.doi.org/10.21786/bbrc/13.11/26 varied behaviour (NCI 2005, Yang et al 2003). The study of Gene expression using microarrays is an important area in the field of biomedical engineering. Latest improvements in DNA microarray technology expedited analysing and assessing the representation levels of a thousands of genes at identical time, so permitting a large generation of microarray data (Fan et al 2009).

Microarray techniques have been effectively utilized widely in the field of biomedical research since they provide the likelihood of performing huge number of tests on genome patterns (Vilda et al 2006). The gene expressions of the microarray consist of several dimensions. The evaluation of each gene is made possible in a particular environment where various types of cancerous tissues are found (Zheng et al 2006). The classification process of microarray is carried out 
in two stages. The initial stage is to choose a group of important and appropriate genes and the next stage is to construct a classification model with which data can be predicated with a higher accuracy. For a precise diagnosis and curing of the cancer disease, it is important to have a good classification model. The presence of immense dimensions in a DNA microarray data poses a problem when used for classifying cancer disease. The reason for this can be the small sample size of DNA micro array when compared to gene size (Du et al 2014).

Normally, a small portion of the genes will be effective for classifying data in a large gene dataset. Hence choosing the appropriate genes plays a significant role in studying microarray data. Also, the correct choice of genes leads to accurate classification of the samples. In case of supervised classification technique, a high-level of precision in data classification can be achieved by selecting appropriate features. Moreover, the technique offers an improved classification quality and reduced computational complexity of the chosen algorithm. The dimensionality of the classification technique can be decreased by obtaining the smallest number of features out of the actual features thus leading to an accurate classification of data. By performing this, inappropriate and unneeded features can be eliminated.

The proposed work challenges the classification of cancer disease with the help of gene expression summaries. An innovative method of examining the microarray dataset to classify cancer is presented in this paper. The novel method initially used Information Gain for selecting features and then uses PCA for reduction of features and ultimately uses SVM for classifying cancer disease. The proposed method enhances the precision of cancer classification by lowering tohe feature count. The performance of the recommended methodology is assessed by applying it on CNS cancer dataset. The classification outcomes are matched with the findings obtained from other recent approaches.

\section{MATERIAL AND METHODS}

Literature Review: The cancer disease can be diagnosed by detecting it at an early stage with the help of a new technique called microarray gene expression. The extremely important genes which may be the root cause of disease can be detected easily by classifying the gene expression data. The classified data helps in treating the cancer patients in an effective way. Feature selection helps in dimensionality reduction and redundancy elimination of the data in an efficient manner during the process of classification. Tan et al. (2008) presented a feature selection framework by combining GA and other methods of feature selection. It was concluded that hybrid approaches prove to be more useful and stronger when compared to applying individual algorithms or techniques on the dataset.

Alexander Statnikov et al (2005) has developed a reliable cancer investigative style centred on microarray data. To offer the model with the ideal blend of classifier, the researcher has used cross validation and genre methods. Also, a detailed evaluation of many algorithms was performed. A novel mixture of feature selection or extraction with Naïve Bayes was used by Rabia Aziz et al (2015) to classify the cancer in huge number of attributes in microarray data. Some of the pre-processing methods like ICA and filtering technique used for extraction of an attributes. Luque-Baena et al.,(2013) have used two approaches namely genetic algorithm and stepwise forward selection for analysis of microarray data. The genetic algorithm approach mixes the mutual information and classification techniques to foresee the cancer disease in patients.

Ding and Peng (2005) has recommended a minimum redundancy maximum relevance (MRMR) feature selection framework to get rid of redundancies observed in microarray gene expression data. Huang and Chow (2005) presented a valuable feature selection proposal by assessing the mutual information built on a supervised data compression algorithm. Zhang et al. (2009) utilized mutual information for multi-label classification and confirmed that this technique enhanced the performance of multilabel classifiers.

Proposed Approach: The nearness of numerous inconsequential and insignificant highlights debases the quality of the investigations of illnesses like cancer. To counter this, it is fundamental to dissect the dataset from the correct point of view. This segment presents an approach for classifying microarray information, which comprises of two stages: i) Feature Selection and Reduction and ii) Classification. The suggested strategy is the combination of IG, PCA and SVM classifier. The point by point portrayal of the proposed strategy is as takes after:

Feature Selection Method using Information Gain: The proposed procedure utilizes IG to select the basic highlights (incorporate assurance (FS)) from the input plans of the quality microarray dataset. Information Choose up (IG) is an entropy-based highlight assessment strategy, broadly utilized inside the field of machine learning. As Information Choose up is utilized in highlight assurance, it is characterized as the whole of information given by the highlight things for the substance category. Information choose up is calculated by how much of a term can be utilized for classification of information, to degree the importance of lexical things for the classification.

$$
\operatorname{Info}(D)=-\sum_{i=1}^{m} p_{i} \log _{2}\left(p_{i}\right)
$$

In the above equation, pi pi is the nonzero likelihood that an self-assertive tuple in Dataset $\mathrm{D}$ belongs to class $\mathrm{C}_{\mathrm{i}}$ and is estimated by $\left|\mathrm{c}_{\mathrm{i}}, \mathrm{D}\right| /|\mathrm{D}|$.

$$
I n f o_{A}(D)=\sum_{j=1}^{v} \frac{\left|D_{j}\right|}{|D|} \times \operatorname{Info}\left(D_{j}\right) .
$$


The term |Dj|/|D | acts as the strength of the jth segment. Data pick up is characterized as the distinction between the first data prerequisite (i.e., based on fair the extent of classes) and the modern prerequisite (i.e., obtained after dividing on A). That is,

$$
\operatorname{Gain}(A)=\operatorname{Info}(D)-\operatorname{Info}_{A}(D)
$$

Choose the highest Gain value and add it to selection set, and repeat this process until the needed attributes are selected.

\section{Attribute Reduction using Principle Component Analysis}

(PCA): PCA looks for $\mathrm{k} n$-dimensional occasions that can best be utilized to speak to the information, where $\mathrm{k}<=\mathrm{n}$. The initial information is hence anticipated onto a much littler space, coming about in dimensionality lessening. PCA points to discover the headings of most extreme change in high-dimensional information and ventures it onto an unused subspace with equal or fewer measurements than the initial one. The calculation for PCA are as takes after:

1. Normalize the $\mathrm{N}$ number of attributes in microarray CNS dataset.

2. Compute the covariance matrix from normalized dataset

3. Find out the eigenvectors (foremost component) and eigenvalues by decomposing the covariance matrix.

4. Sort the eigenvalues by eigenvectors.

5. Select $\mathrm{k}$ eigenvectors which compare to the $\mathrm{k}$ biggest eigenvalues, where $\mathrm{k}$ is the dimensionality of the modern include subspace $(\mathrm{k} \leq \mathrm{d})$.

6. Build a projection framework $\mathrm{W}$ from the largest $\mathrm{k}$ eigenvectors.

7. Change the $\mathrm{N}$ attribute input CNS dataset $\mathrm{X}$ utilizing the projection framework $\mathrm{W}$ to get the new $\mathrm{k}$-attribute include subspace.

Classification using Support Vector Machine: The SVM may be a state-of-the-art classification strategy presented in 1992 by Boser. The hypothesis of SVM is based on the thought of auxiliary risk minimization. The microarray data set has been classified as a two class problem where the two classes are cancer and normal. To begin with, let vector $x \in R^{n}$ indicate a design to be classified, and let scalar y signify its category name (i.e., $y \in\{ \pm 1\}$ ).

Additionally, let $\left\{\left(x_{i}, y_{i}\right), i=1,2, \ldots, n\right\}$ indicates the set of $\mathrm{n}$ number of samples for training, where every record in the dataset has a known class labelled as . The matter is to work out a classifier $\mathrm{f}(\mathrm{x})$ (i.e., a choice work) that will appropriately classify an input design. After applying the Information Gain and PCA from the CNS dataset, the classification technique called SVM is applied to classify the given dataset into cancer and normal.

\section{RESULTS AND DISCUSSION}

The comes about gotten utilizing our proposed calculation on the CNS microarray dataset is examined in a successive way. The CNS dataset presents the resulting forecast of the patients for embryonal tumors. This contains an add up to of 60 tests ( 21 are survivors and 39 are disappointments) with 7129 number of genes. This is often utilized for 2-class classification issue. A combination of techniques like information Gain, Principal Component Analysis and SVM were used to classify data and also a comparison was drawn with the results obtained with K-nearest-neighbors (KNN) and Decision Tree Algorithms.

Table 1. Classification Results using CNS dataset with all features

\begin{tabular}{|l|c|c|c|l|}
\hline Classifier & $\begin{array}{c}\text { Classification } \\
\text { Accuracy }\end{array}$ & AUC & Precision & Recall \\
\hline kNN & 0.600 & 0.577 & 0.563 & 0.600 \\
\hline Decision Tree & 0.567 & 0.579 & 0.617 & 0.567 \\
\hline SVM & 0.650 & 0.217 & 0.423 & 0.650 \\
\hline
\end{tabular}

Table 2. Classification Results using CNS dataset with selected features - Information Gain

\begin{tabular}{|l|c|c|c|c|}
\hline Classifier & $\begin{array}{c}\text { Classification } \\
\text { Accuracy }\end{array}$ & AUC & Precision & Recall \\
\hline kNN & 0.783 & 0.875 & 0.793 & 0.783 \\
\hline Decision Tree & 0.800 & 0.752 & 0.797 & 0.800 \\
\hline SVM & 0.833 & 0.875 & 0.838 & 0.833 \\
\hline
\end{tabular}

Table 3. Classification Results using CNS dataset with PCA

\begin{tabular}{|l|c|c|c|c|}
\hline Classifier & $\begin{array}{c}\text { Classification } \\
\text { Accuracy }\end{array}$ & AUC & Precision & Recall \\
\hline kNN & 0.583 & 0.558 & 0.521 & 0.583 \\
\hline Decision Tree & 0.617 & 0.512 & 0.596 & 0.617 \\
\hline SVM & 0.633 & 0.529 & 0.419 & 0.633 \\
\hline
\end{tabular}

Table 4. Classification Results using CNS dataset with combination Information Gain and PCA

\begin{tabular}{|l|c|c|c|c|}
\hline Classifier & $\begin{array}{c}\text { Classification } \\
\text { Accuracy }\end{array}$ & AUC & Precision & Recall \\
\hline kNN & 0.783 & 0.875 & 0.793 & 0.783 \\
\hline Decision Tree & 0.617 & 0.563 & 0.604 & 0.617 \\
\hline SVM & 0.878 & 0.896 & 0.870 & 0.873 \\
\hline
\end{tabular}


The performance of the classifier was evaluated using classification accuracy, precision, Recall, ROC curve and Area Under the Curve (AUC). To illustrate the adequacy and practicality of the proposed strategy, the comes about of the other classification strategies are too displayed in Tables 1 to 4 .

In Table 1, the CNS microarray data are classified by SVM, Decision Tree and KNN right away with all the features. The features selected by Information Gain and PCA was applied in the data set. The Table 2 highlight the experimental results. In Table 4, the combination of Information Gain, PCA and different classification techniques are applied in the dataset. It can be seen from Tables $1-4$, the IG+ PCA+SVM perform better than other individual and combinational methods, which demonstrates the effectiveness of the proposed approach. It is evident from the proposed method that the classification accuracy is high with SVM classifiers. Accurate results with reduced variation of classification performance was obtained when compared to other gene selection techniques. Thus, the proposed method displays improved classification of CNS microarray data. The performance comparison of several other methods with the proposed method using ROC Curve can be inferred from Fig. 1-4.

Figure 1: AUC curve - Classification Results using CNS dataset with all features

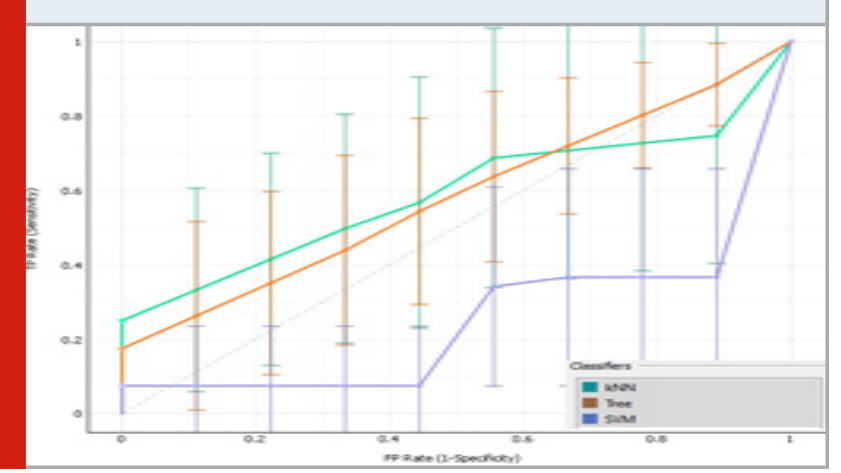

Figure 2: AUC Curve - Classification Results using CNS dataset with selected features - Information Gain

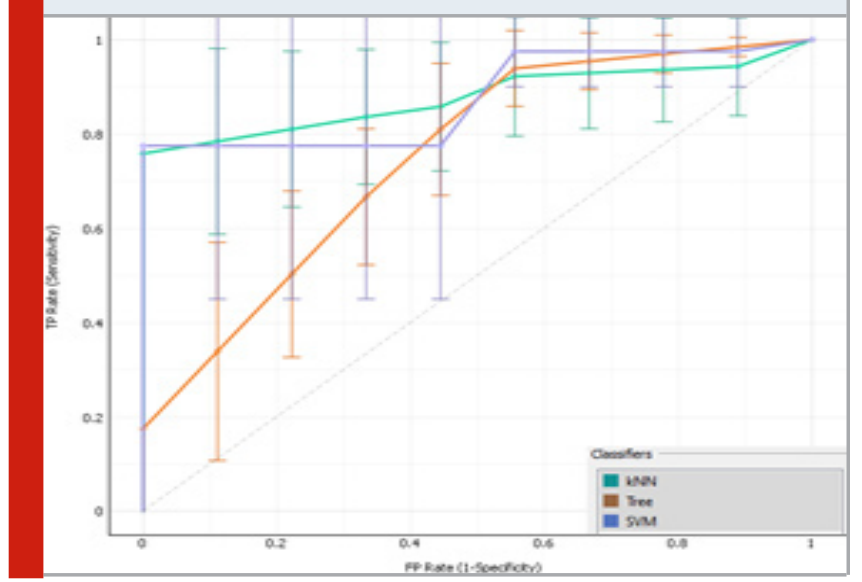

Figure 3: AUC Curve - Classification Results using CNS dataset with PCA

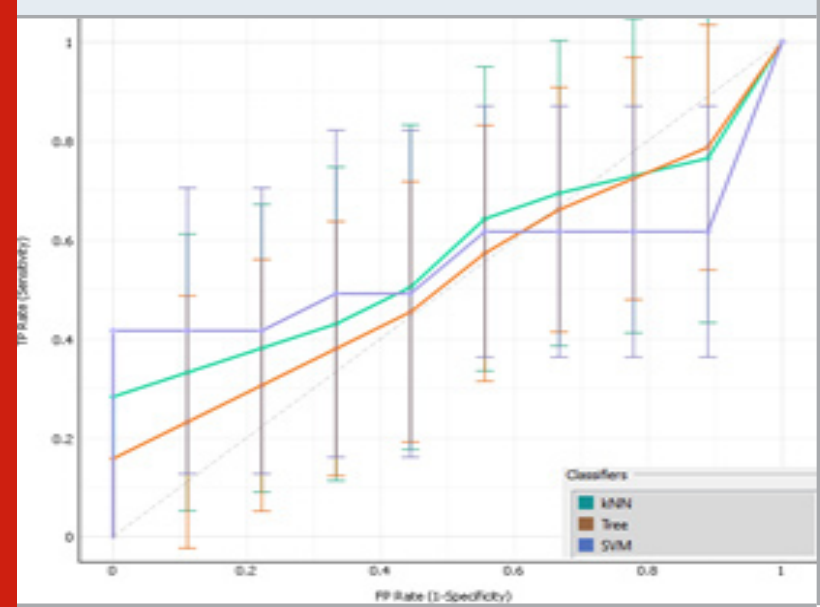

Figure 4: AUC Curve - Classification Results using CNS dataset with combination Information Gain and PCA

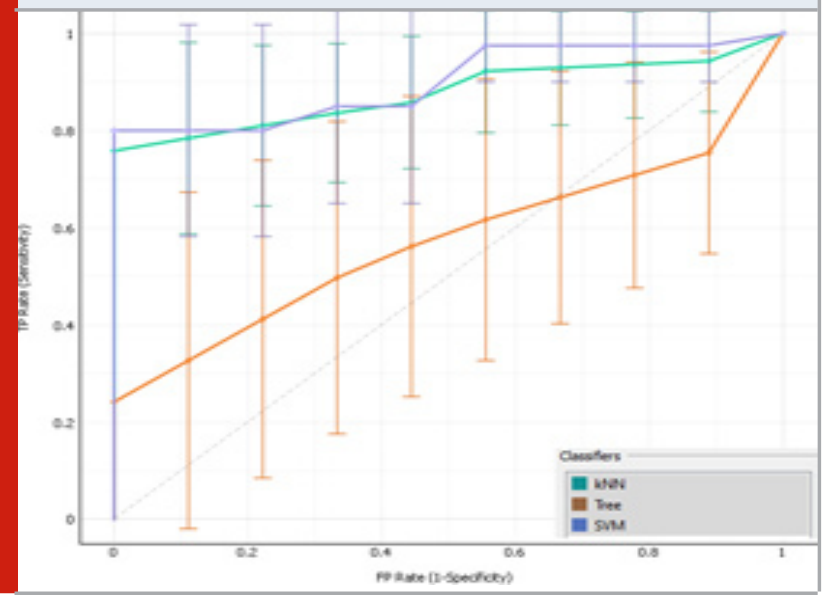

The graphical representation of the classification accuracy of CNS cancer dataset with various genes selected utilizing Information Gain, PCA with SVM, KNN and Decision Tree classifiers were shown in figure 4. The peak in the graph indicates highest classification accuracy of the dataset after applying the proposed method.

\section{CONCLUSION}

In the field of medical investigation, gene selection is a great concern for the researchers while predicting disease in patients. Proper selection of genes contributes to the performance improvement of the classifier model in terms of quality, accuracy, and complexity. This paper presents a various feature selection method and classification techniques applied in CNS data set. In the proposed work, ROC displays the finest subset of genes thus offering a good accuracy of classification of CNS data set. The investigational results prove that the proposed combination of gene selection method and PCA along with SVM provides improved results 
when compared to other techniques. Based on the investigational findings, the suggested approach displays a good performance accuracy by using smaller number of records and selected genes.

Conflicts of Interest: The authors declare no conflicts of interest.

\section{REFERENCES}

Alexander Statnikov, Constantin F. Aliferis, Ioannis Tsamardinos, Douglas Hardinand Shawn Levy (2005), "A comprehensive evaluation of multicategoryclassification methods for microarray gene expressioncancer diagnosis”, Bioinformatics, Vol. 21, No. 5, pp 631643.

Ding C, H. Peng (2005), Minimum redundancy feature selection from microarray gene expression data, J. Bioinf. Comput. Biol. 3 (02) , 185-205.

Du D, K. Li, X. Li, M. Fei (2014), A novel forward gene selection algorithm for microarray data. Neurocomputing 133, 446-458.

Fan L, K.-L. Poh, P. Zhou (2009), A sequential feature extraction approach for Naïve Bayes classification of microarray data. Expert Syst. Appl. 36, 9919-9923.

Huang D, T.W. Chow (2005), Effective feature selection scheme using mutual information, Neuro computing 63, 325-343.

Luque-Baena R.M, D. Urda, J.L. Subirats, L. Franco, and J.M. Jerez (2013), "Analysis of Cancer Microarray Data using ConstructiveNeural Networks and Genetic Algorithms", IWBBIO, Proceedings, Canada, pp. 55-
63.

NCI Brain Tumor Progress Review Group (2005) ; "Report", http.//accessible.ninds.nih.gov/find-people/ groups/brain-tumor-prg/BT PRGReport.htm, February, 2005.

Rabia Aziz, C.K. Verma and Namita Srivastav (2015) , "A Weighted-SNR Feature Selection from Independent Component Subspace for NB Classification of Microarray Data”, International Journal of Advanced Biotechnology and Research, Vol. 6, Issue 2, pp. 245-255.

Tan F, X. Fu, Y. Zhang (2008), A.G. Bourgeois, A genetic algorithm-based method for feature subset selection, Soft Comput. 12 (2), 111-120.

Vilda P.G. , F. Díaz, R. Martínez, R. Malutan, V. Rodellar, C.G. Puntonet (2006), Robust preprocessing of gene expression microarrays for independent component analysis. Independent Component Analysis and Blind Signal Separation, Springer, pp. 714-721.

Yang Y, S Guccione, MD Bednarski (2003), ”Comparing genomic and histologic correlations to radiographic changes in tumors: A murine SCC Vll model study", Academic Radiology, vol. 10, issue. 10, pp. 11651175.

Zhang M L, J.M. Peña, V. Robles (2009), Feature selection for multi-label naive Bayes classification, Inf. Sci. 179 (19), 3218-3229.

Zheng C H, D.-S. Huang, L. Shang (2006), Feature selection in independent component subspace for microarray data classification. Neurocomputing 69, 2407-2410. 